\title{
Asteroseismology of $\delta$ Scuti stars in open clusters: Praesepe
}

\author{
J. C. Suárez ${ }^{1,2}$, E. Michel ${ }^{2}$, G. Houdek ${ }^{3}$, F. Pérez Hernández ${ }^{4,5}$ and \\ Y. Lebreton ${ }^{6}$ \\ ${ }^{1}$ Instituto de Astrofsica de Andaluca (CSIC), CP3004, Granada, Spain ${ }^{2}$ LESIA, \\ Observatoire de Paris-Meudon, UMR8109, France \\ ${ }^{3}$ Institute of Astronomy, University of Cambridge, Cambridge CB30HA, UK \\ ${ }^{4}$ Instituto de Astrofísica de Canarias (IAC), Tenerife, Spain \\ ${ }^{5}$ Departamento de Astrofísica, Universidad de La Laguna, Tenerife, Spain \\ ${ }^{6}$ GEPI, Observatoire de Paris-Meudon, Meudon, France
}

\begin{abstract}
The present paper provides a general overview of the asteroseismic potential of $\delta$ Scuti stars in clusters, in particular focusing on convection diagnostics. We give a summarise of the last results obtained by the authors for the Praesepe cluster of which five $\delta$ Scuti stars are analysed. In that work, linear analysis is confronted with observations, using refined descriptions for the effects of rotation on the determination of the global stellar parameters and on the adiabatic oscillation frequency computations. A single, complete, and coherent solution for all the selected stars is found, which lead the authors to find important restrictions to the convection description for a certain range of effective temperatures. Furthermore, the method used allowed to give an estimate of the global parameters of the selected stars and constrain the cluster.
\end{abstract}

\section{Introduction}

The main idea of the work here outlined is to compare ranges of observed and predicted unstable modes and analyse them in terms of ranges of radial modes. That methodology was conceived and used in Michel et al. (1999), hereafter M99, and here is revisited. The use of radial modes is justified because driving and damping in $\delta$ Scuti stars takes place predominantly in the He II ionisation zone, which is rather close to the stellar surface, where the vertical 
scale is much less than the horizontal scale of the oscillations and when $\ell$ is low the modal inertia is quite insensitive to degree $\ell$. We consider the following stars belonging to the Praesepe cluster: BW Cnc, BS Cnc, BU Cnc and BN Cnc (already included in the sample considered by M99), which were observed by several campaigns of the STEPHI network (Michel et al. 1995), and a fifth star, BV Cnc, which was observed by Frandsen et al. (2001).

The present work intends to search for one particular solution that explains the whole set of observations, instead of sets of individual solutions for each star (methodology followed by M99 whose results are considered here as the reference domain of possible solutions). To do so, refined techniques for modelling intermediate mass stars are required, in particular those taking different effects of rotation into account. This requirement is fulfilled in this work with modern techniques that largerly improve those adopted in M99 and other precedent works. One of the major improvements is the use the complete second-order formalism (including near degeneracy effects) developed by Suárez et al. (2006) based on the works by Dziembowski \& Goode (1992) and Soufi et al. (1998), which, in addition, takes the effect of the star deformation due to rotation into account.

\section{Procedure}

Firstly, we calculate the fundamental parameters of each selected star. To do so we apply the method by Pérez Hernández (1999) which corrects the photometric observables for the effect of rotation. That method is model-dependent, so that solutions are obtained by adjusting some typically free parameters. The best solution given by the correction for the effect of rotation was obtained for $\alpha_{\mathrm{MLT}}=1.614$ and $d_{\mathrm{ov}}=0.2$. This best solution correspond to an age of the cluster of $650 \mathrm{Myr}( \pm 20-40 \mathrm{Myr})$. The age uncertainty of 20-40 Myr can be neglected in terms of global characteristics of the non-rotating co-partners (see the influence of age on photometric corrections for rotation in different open clusters in Suárez et al. 2002).

The fundamental parameters obtained are then used to build representative asteroseismic models for each star, consisting of pseudo-rotating equilibrium models and their corresponding adiabatic oscillation spectra. Theoretical adiabatic oscillation spectra are computed with the oscillation code FILOU (Tran Minh \& Lon 1995, Suárez 2002) which uses the complete treatment of secondorder effects of rotation by Suárez et al. (2006), based on the formalisms by Dziembowski \& Goode (1992) and Soufin et al (1998). These asteroseismic models are then used to determine the observed radial orders ranges, which, as it was done in $\mathrm{M} 99$, are then confronted with mode instability predictions obtained from a linear stability analysis. 
Unstable radial modes are calculated by means of linear stability computations which are carried out in the manner of Balmforth (1992). We adopt the nomenclature of $\mathrm{M} 99$, i.e. $\alpha_{\mathrm{NL}}=l / H_{\mathrm{p}}$ is the mixing-length parameter of the non-local convection model used in the stability computations. The non-local mixing-length parameter $\alpha_{\mathrm{NL}}$ is calibrated to the same depth of the outer convection zone as suggested by the evolutionary computations which use the standard mixing-length formulation by Böhm-Vitense (1958) and a local mixing-length parameter $\alpha_{\mathrm{MLT}}=1.614$, for which the equivalent calibrated value of $\alpha_{\mathrm{NL}}=1.89$ is obtained. As in M99, a second series of stellar models with $\alpha_{\mathrm{NL}}=1.50$ in order to analyse the impact of varying the mixing-length on mode stability.

\section{Comparison between observed and predicted ranges of unstable ra- dial modes}

We compare ranges of observed and predicted radial orders $n$ of unstable modes for two values of $\alpha_{\mathrm{NL}}$ : 1.89 and 1.50. This comparison is shown in Fig. 1 in which an uncertainty of $\pm 1 n$ in the determination of the range of radial orders (see Suárez et al. (2007) for a detailed explanation of uncertainty sources). For each star, we have two representative models correspondig to considering $\pm 10 \%$ of the observed $V \sin i$ value, so we calculate four radial order ranges are calculated per object. It is found that, in general, the observed ranges are in good agreement with the theoretical predictions using $\alpha_{\mathrm{NL}}=1.50$, whereas with $\alpha_{\mathrm{NL}}=1.89$ the predicted ranges are in grave disagreement with the observations for the intermediate mass stars BW $\mathrm{Cnc}$ and $\mathrm{BS} \mathrm{Cnc}$, which present a temperature range of $\log T_{\text {eff }}=3.87-3.88$. For these stars a smaller mixinglength parameter $\alpha_{\mathrm{NL}}$ is then required than suggested from a calibrated solar model. The need of a smaller value for $\alpha_{\mathrm{NL}}$ than that from a calibrated solar model was also reported by Daszyńska-Daszkiewicz et al. (2005) for the $\delta$ Scuti star FG Vir. For the massive objects, BU Cnc and BN Cnc, the predicted unstable ranges are compatible with the observed results $( \pm 1 n)$, for both $\alpha_{\mathrm{NL}}=1.50$ and $\alpha_{\mathrm{NL}}=1.89$. The results for both objects are thus not sensitive to the value of $\alpha_{\mathrm{NL}}$. This is to be expected because these more massive stars have shallower outer convection zones and thus their structures are less sensitive to the assumed value of the mixing-length parameter. Finally, for the less massive object, BV Cnc, the values for $\alpha_{\mathrm{NL}}$ cannot be distinguished. Nevertheless, the observed ranges agree with the theoretical predictions within $\pm 1 n$. Therefore, in general, these results constitute a consistent solution in terms of physics and cluster membership, and the observed and theoretical ranges of radial orders are in reasonable agreement for all the stars considered in this work.

Details of the procedure, model characteristics, as well as the detailed com- 

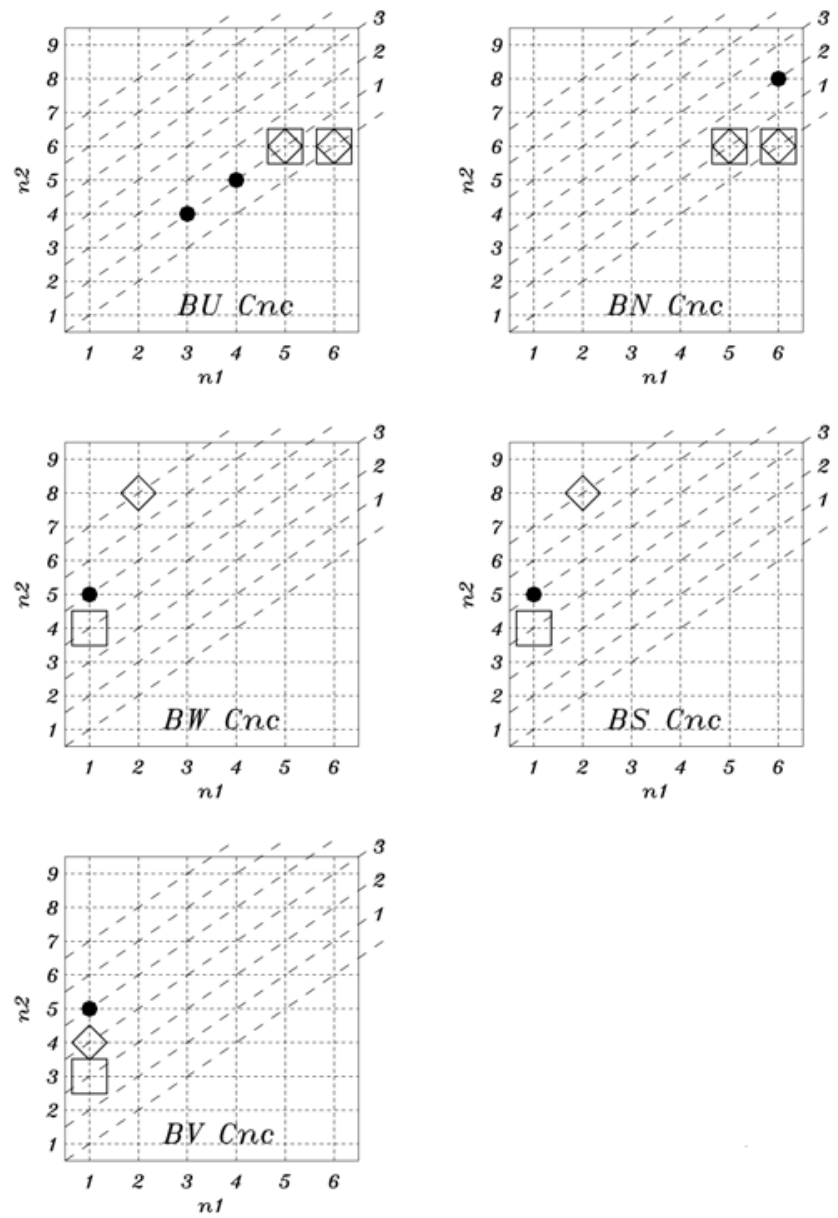

Figure 1: Observed and predicted (using linear stability analysis) ranges of unstable radial modes for the selected $\delta$ Scuti stars $\left(n_{1}\right.$ is the lowest value, and $n_{2}$ the largest value of the radial order of the unstable modes) displayed for the selected $\delta$ Scuti stars. Filled circles represent the observed ranges. Rhombus and squares correspond to predicted radial order ranges for $\alpha_{\mathrm{NL}}=1.89$ and $\alpha_{\mathrm{NL}}=1.50$, respectively. Each diagonal-dashed line represents the width (in radial orders) of the represented ranges. Taken from Suárez et al. (2007) 
parison between the present results with those of M99 are given in a forthcoming paper Suárez et al. (2007). We note that, as in M99, the instability predictions are carried out using equivalent envelope models which do, however, not take the effect of rotation into account. This is so because, up to date, there are no reliable theories available which describe the effect of rotation on mode stability. Nevertheless, in a forthcoming paper Suárez et al. (2007), a crude estimate of this effect is addressed, which assumes that mode stability depends predominantly on the effective temperature of the model (Pamiatnykh 1975). As well, in that work, more details on the procedure, the characteristics of models, and a detailed comparison between the present results with those of M99 are provided.

Acknowledgments. JCS acknowledges support by the Instituto de Astrofísica de Andalucía by an I3P contract financed by the European Social Fund and from the Spanish Plan Nacional del Espacio under project ESP200403855-C03-01. GH acknowledges support by the Particle Physics and Astronomy Research Council of the UK.

\section{References}

Böhm-Vitense, E. 1958, ZA 46, 108

Balmforth, N. J. 1992, MNRAS255, 603

Daszyńska-Daszkiewicz, J., Dziembowski, W. A., Pamyatnykh, A. A., et al. 2005, A\&A 438, 653

Dziembowski, W. A., \& Goode, P. R. 1992, ApJ 394, 670

Frandsen, S., Pigulski, A., Nuspl, J., et al. 2001, A\&A 376, 175

Michel, E., Chevreton, M., Belmonte, J. A., et al. 1995, in Hoeksema, J., Domingo, V., Fleck, B., Battrick, B., eds, 4th Soho Workshop, ESA SP-376, p. 533

Michel, E., Hernández, M. M., Houdek, G., et al. 1999, A\&A 342, 153

Pamiatnykh, A. A. 1975, in Sherwood, V. E., Plaut, L. eds, IAU Symp. 67. Variable Stars and Stellar Evolution. Dordrecht, Reidel Publishing, pp. 247

Pérez Hernández, F., Claret, A., Hernández, M. M., \& Michel, E. 1999, A\&A 346, 586

Soufi, F., Goupil, M. J., Dziembowski, W. A. 1998, A\&A 334, 911

Suárez, J. C. 2002, Ph.D. Thesis, ISBN 84-689-3851-3, ID 02/PA07/7178

Suárez, J. C., Goupil, M. J., \& Morel, P. 2006, A\&A 449, 673

Tran Minh, F., \& Lon, L. 1995, Physical Process in Astrophysics 219 